\title{
Esophageal Infection, CTCAE
}

National Cancer Institute

\section{Source}

National Cancer Institute. Esophageal Infection, CT CAE. NCI Thesaurus. Code C143454.

A disorder characterized by an infectious process involving the esophagus. 